\title{
Latest Advances on Serial Crystallography at XFELs and Synchrotron Sources
}

\author{
Jose Martin-Garcia1 ${ }^{1}$, John Spence ${ }^{1,3}$, Petra Fromme 1,2
}

1. Center for Applied Structural Discovery, Biodesign Institute, Arizona State University, Tempe, AZ, USA

2. School of Molecular Sciences, Arizona State University, Tempe, AZ, USA

3. Department of Physics, Arizona State University, Tempe, AZ, USA

EMail Contact: jose.martingarcia@asu.edu

X-ray Free Electron lasers (XFELs) produce extraordinarily powerful X-rays to take snapshots of molecules as they move, with the potential to make molecular movies and discover how they work. The groundbreaking serial femtosecond crystallography (SFX) experiment was conducted at the LCLS XFEL ten years ago'. Since then, new technologies developed at XFELs and their implementation in the field of SFX has opened up a new era in structural biology. As a result, the field is quickly expanding, allowing structural biologists access to previously restricted scientific areas ${ }^{2}$. This emerging field has also cultivated new methods for crystallization, and assessment of nano/microcrystals, sample delivery, and data processing².

In addition to its use at XFELs, the serial crystallography (SX) approach can also be done at synchrotron radiation sources and have gained popularity in the past few years up to the point that it can now be a viable alternative to scarce $X$-ray free electron laser sources. More recently, pink beam serial crystallography has come into play and demonstrating that crystallographic structures of proteins can be obtained from small crystals by just using a few hundred snapshots ${ }^{3,4}$. Monochromatic and pink beam experiments have demonstrated the feasibility of serial data collection using micro-crystals at numerous microfocus beamlines at the most powerful synchrotron radiation sources in the world. Upcoming developments in beamline optics, detector technology and synchrotron sources by itself will enable the use of even smaller micro-crystals $(<1 \mu \mathrm{m})$, the use of larger macromolecules as well as the possibility of conducting mix-and-inject time-resolved studies.

In my talk I will be presenting the newest and more exciting advances achieved by the SX technology at XFELs and at synchrotron radiation sources. As well, I will be mentioning about the current and upcoming more powerful XFELs as well as the new technology in the form of new type of compact XFEL. Compact XFELs could revolutionize the field by making the technology available to many more scientists around the world and accelerate the rate at which we discover knowledge for helping solve critical global challenges.

References:

[1] Chapman HN, Fromme P, Barty A, et al. Femtosecond X-ray protein nanocrystallography. Nature. 2011;470(7332):73-7.

[2] Martin-Garcia JM, Conrad CE, Coe J, Roy-Chowdhury S, Fromme P. Serial femtosecond crystallography: A revolution in structural biology. Arch Biochem Biophys. 2016;602:32-47.

[3] Meents A, Wiedorn MO, Srajer V, et al. Pink-beam serial crystallography. Nat Commun. 2017;8(1):1281.

[4] Martin-Garcia JM, Zhu L, Mendez D, et al. High-viscosity injector-based Pink Beam Serial Crystallography of Micro-crystals at a Synchrotron Radiation Source. IUCrJ. 2019 (accepted) 